Trauma Berufskrankh 2007 - 9[Suppl 3]: S297-S301

DOI 10.1007/s10039-007-1287-3

Online publiziert: 9. September 2007

(c) Springer Medizin Verlag 2007

M. Müller · T. Mückley · G.O. Hofmann

Klinik für Unfall-, Hand- und Wiederherstellungschirurgie,

Friedrich-Schiller-Universität, Jena

\title{
Kosten und Komplikationen der Materialentfernung
}

tischen, aber auch bei asymptomatischen Patienten bedacht werden. So klagten z. B. Patienten, die vor der Implantatentfernung asymptomatisch waren, nach Entfernung eines Tibianagels in 12\% über Knieschmerzen [1], in einer anderen Studie gaben $20 \%$ der Patienten Schmerzen nach der Entfernung von Ober- oder Unterschenkelnägeln an $[10,11]$. Da bei asymptomatischen Patienten eine Verschlechterung, bei symptomatischen $\mathrm{Pa}$ tienten nach der Entfernung von Implantaten aber eine häufige Besserung der Symptome erreicht werden können (bis $\mathrm{zu} 78 \%$ der Fälle [10]), wird von mehreren Autoren nur die Materialentfernung bei symptomatischen Patienten empfohlen $[1,4,10,11,17]$.

Weitere Indikationen zur Implantatentfernung können die möglichen bekannten Risiken bei Belassen der Implantate sein:

- späte hämatogene Infektionen [14],

- Metalltoxizität,

- Metallallergenisierung [7],

- Korrosion $[5,8]$ sowie

- implantatbedingte Osteopenie und

- Kanzerogenität $[15,25]$.

Ermüdungsbruch am Implantatende, erschwerte Diagnostik (z. B. MRT) und erschwerte Implantatentfernung nach langer Liegezeit [23] sind weitere Gefahren.

\section{Kontra}

Neben den anerkannten Indikationen zur Implantatentfernung sind auch Gründe für deren Belassen benannt worden:

- fehlende relevante klinische Symptome oder Komplikationen,

- fortgeschrittenes Lebensalter,
- Metallimplantate im Bereich der oberen Extremität (besonders Platten am Humerusschaft),

- einzelne Schrauben und Implantate aus Titan.

Ebenso können bei einer operativen Metallentfernung abgebrochene Metallteile oder verbliebene Unterlegscheiben belassen werden [24].

\section{Komplikationen}

\section{Allgemeine Risiken einer Operation}

Sie gelten natürlich auch für die Implantatentfernung, wie Narkoserisiko, Blutung und Hämatombildung, postopera-

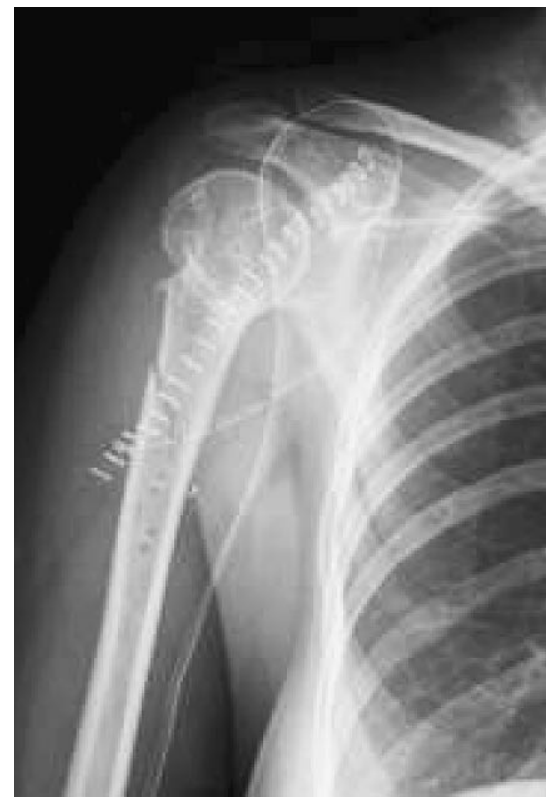

Abb. $1 \Delta$ Fraktur der Oberarmschafts nach Implantatentfernung einer Platte am proximalen Humerus 


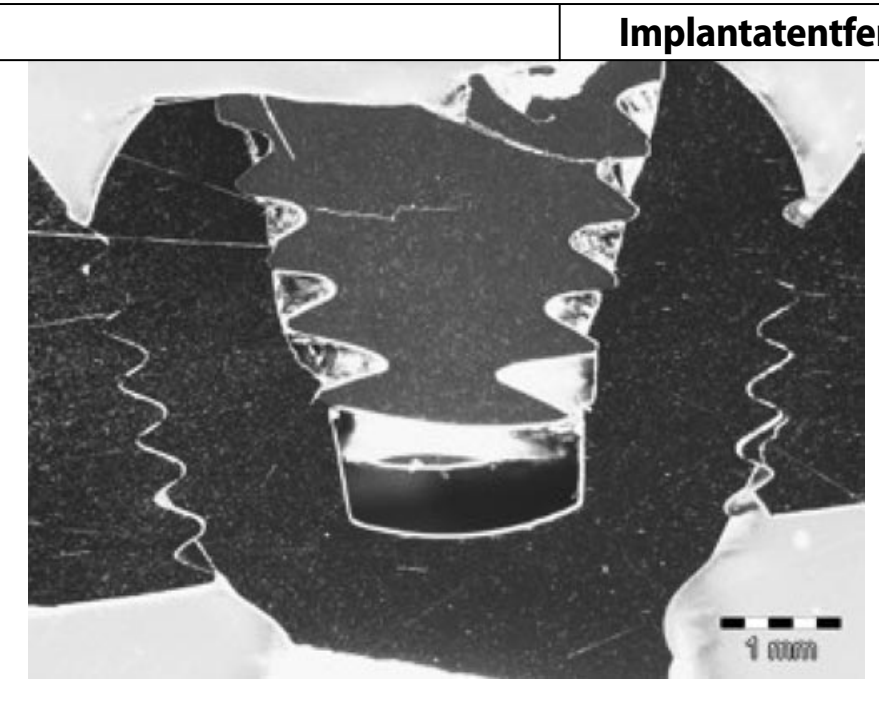

Abb. $2<$ Abgebrochene Extraktionsschraube im Schraubenkopf eines winkelstabilen Implantats, Schraubenkopf weiterhin fest im Plattenloch verankert

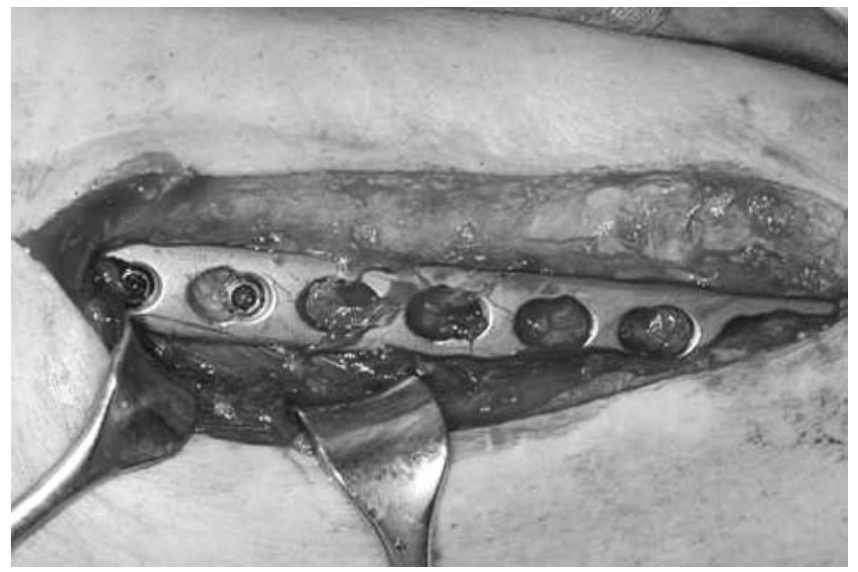

Abb. $3<$ Zerstörter Inbus einer winkelstabilen Schraube (LCP)

tive Infektion und Wundheilungsstörung, Thrombose und Embolie.

Infektionen treten bei Routineimplantatentfernungen in bis $\mathrm{zu} 11 \%[4,10]$ bis $14 \%$ [21] der Fälle auf, bei initial offenen Frakturen sogar in bis zu $43 \%$ der Fälle [21].

\section{Spezielle Komplikationen}

Neben den allgemeinen Risiken und Komplikationsmöglichkeiten sind v. a. die typischen Risiken der Implantatentfernung $\mathrm{zu}$ beachten. Insbesondere bei den relativen Indikationen und den asymptomatischen Patienten muss eine genaue Aufklärung über die z. T. gravierenden $\mathrm{Ri}$ siken erfolgen.

In Abhängigkeit von der Lokalisation sind es v. a. Nervenläsionen und peri- oder postoperative Refrakturen sowie in Abhängigkeit vom verwendeten Implantat die unvollständige Materialentfernung. Weiterhin muss eine mögliche Ausweitung der Operation (Reosteosynthese, erweitere Zugänge) mit einkalkuliert/geplant werden.
Nervenläsion. In der Gruppe der speziellen Komplikationen gehört sie zu den schwerwiegenden und häufigen Komplikationen der Implantatentfernung. Aufgrund der Anatomie und der Zugangswege sind v. a. folgende Nerven betroffen:

- der N. radialis beim dorsalen Zugang zur Plattenosteosynthese des Humerusschafts,

- der N. axillaris beim Deltoideus-SplitZugang bei proximalen Humerusfrakturen,

- der motorische Ast der Nn. radialis und medianus bei der Plattenosteosynthese von Radiusfrakturen,

- der N. ischiadicus beim dorsalen Zugang zum Hüftgelenk und

- der N. peroneus superficialis am Unterschenkel.

Die Anzahl der Nervenschädigungen bei Implantatentfernungen wird selten publiziert. Über eine Schädigung nervaler Strukturen von $12 \%$ bei Implantatentfernungen nach Unterarmfrakturen wurde berichtet $[21]$.
Postoperative Refrakturraten. Sie sind für verschiedene Körperregionen, aber v. a. für Unterarmfrakturen, bekannt (- Abb. 1). Refrakturraten nach Implantatentfernung am Unterarm betragen in publizierten Serien bis zu 19\% [6] bzw. $30 \%$ [13]. Aber auch intraoperative Frakturen wie der Bruch eines Röhrenknochens durch Rückschlagen des Marknagels bei belassenem Verrieglungsbolzen sollten erwähnt werden $[1,11]$.

\section{Unvollständige Implantatentfernung.}

Sie muss als weitere typische Komplikation genannt werden. Sie kann verschiedene Gründe haben:

- Eine vollständige Materialentfernung wäre nur mit nicht zu vertretendem Aufwand möglich (z. B. Eröffnen der Markhöhle bei intraossärer Kirschner-Draht-Lage, Überbohren abgebrochener Schrauben oder Ähnliches).

- vermeidbare unvollständige Materialentfernungen (z. B. vergessene Unterlegscheiben oder Einzelschrauben)

Um eine unvollständige Materialentfernung zu unterlassen, ist eine Durchleuchtungskontrolle mit dem Bildwandler zu empfehlen. Die Kontrolle mit dem C-Bogen sollte grundsätzlich intraoperativ durchgeführt werden, dabei lassen sich auch weitere Komplikationen, wie periimplantäre Frakturen bei schwieriger Materialentfernung, erkennen und in gleicher Sitzung adäquat behandeln. Die Bildwandlerkontrolle ist zusätzlich erforderlich zur Dokumentation des gewünschten Ergebnisses. Verfügt das System über eine DICOM-Schnittstelle und ist somit zur Archivierung im PACS geeignet, können Zeit und Kosten konsumierende postoperative Röntgenkontrollen bei entsprechender intraoperativer Bildqualität vermieden werden.

Weitere Komplikationen - speziell bei winkelstabilen Implantaten. Bedingt durch die winkelstabilen Implantate kommt es speziell durch die Kopfverriegelungsschrauben zu neuen Komplikationen, die bei nichtwinkelstabilen Implantaten nicht bekannt waren. In entsprechenden Publikationen wurde über Schrauben eines LISS („less invasive sta- 
bilization system"), die nicht auf normalem Weg entfernt werden konnten, mit einer Häufigkeit von $17-100 \%[9,12]$ berichtet. In beiden Fällen mussten spezielle Techniken (konischer Innengewindeschneider und Stahlbohrer) bemüht werden, um dann bei erhöhtem Operationstrauma und verlängerter Operationszeit die Implantate zu bergen ( $\bullet$ Abb. 2).

Publizierte Daten unserer Klinik, in denen wir die Komplikationsraten winkelstabiler und nichtwinkelstabiler Platten verglichen, zeigen bei der Materialentfernung etwa $19 \%$ intraoperative Komplikationen bei den winkelstabilen Implantaten bei fehlenden Komplikationen in der Gruppe der konventionellen Implantate [19]. Gründe für Probleme bei den Kopfverriegelungsschrauben könnten sein:

- ein höheres Drehmoment für die Entfernung im Vergleich zu Stahlimplantaten (bessere Osteointegration der Titanimplantate),

- der Formschluss der Winkelstabilität durch das Platte-Schraubenkopf-Design,

- eine Beschädigung des Innensechskants (- Abb. 3) und

- ein zu hohes Drehmoment beim Einbringen der Schraube.

Das so genannte „Kaltverschweißen“, also die feste Metall-Metall-Verbindung bei Raumtemperatur, wird begünstigt durch die speziellen Materialeigenschaften der chirurgischen Implantate. Bei Werkstoffen mit hoher Oberflächengüte tritt dieses Phänomen häufiger auf, besonders bei galvanisierten Oberflächen. Weiterhin wird diese feste Verbindung gefördert durch Reibung und Druck und durch eine Beschädigung der Oxygenierungsschicht. Durch Oxygenierung von Metallen, die in Abhängigkeit vom gewählten Element durch verschiedene Techniken erreicht wird, kann diese Tendenz zur „Kaltverschweißung “ verringert werden. Sie ist jedoch eher selten, meist handelt es sich um Verklemmungen durch u. a hohen Formschluss, geringe Abweichung in der Einbringungsrichtung der Schrauben usw.

Zusätzlich ist für winkelstabile Implantate gezeigt worden, dass in einigen Fällen das Drehmoment zum Entfernen der Schrauben über dem des (limi-

Trauma Berufskrankh 2007 · 9[Suppl 3]: S297-S301 DOI 10.1007/s10039-007-1287-3

(c) Springer Medizin Verlag 2007

M. Müller ·T. Mückley · G.O. Hofmann

Kosten und Komplikationen der Materialentfernung

\section{Zusammenfassung}

Die Implantatentfernung ist eine der häufigsten orthopädisch-traumatologischen Operationen. Da dabei z. T. schwer wiegende Komplikationen auftreten, sind besonders die relativen Indikationen, $v$. a. bei Beschwerdefreiheit des Patienten, kritisch zu werten. Neben den allgemeinen Komplikationen der Implantatentfernung wie Infektionen sind v. a. typische Komplikationen wie Refrakturen, Nervenläsionen, nach Implantatentfernung neu aufgetretene Schmerzen und unvollständige Implantatentfernung zu nennen. Speziell die winkelstabilen Implantate lassen eine neue Qualität der Komplikationen bei der Entfernung erkennen. Somit kann die Mate- rialentfernung nur bei symptomatischen $\mathrm{Pa}$ tienten empfohlen werden. Sie ist aber in einigen Ländern bereits die zweithäufigste orthopädisch-traumatologische Operation und hat deshalb neben der großen volkswirtschaftlichen Bedeutung eine fallzahl- und, in Abhängigkeit von vielen Variablen, potenziell Erlös steigernde Auswirkung. Bei Abrechnung nach G-DRG kann sie sich Erlös erhöhend auswirken, wenn schnell und komplikationsarm operiert wird.

\section{Schlüsselwörter}

Implantatentfernung · Winkelstabile Implantate $\cdot$ Komplikationen $\cdot$ Kosten $\cdot$ Erlös

\section{Costs and complications of implant removal}

\section{Abstract}

Implant removal procedures are among the most frequently performed orthopaedic operations. Since they can have potentially severe complications, relative indications for such procedures have to be thoroughly considered especially when patients have no symptoms. Apart from such general complications as infection, the possibility of refractures, nerve lesions, pain arising for the first time after surgery and incomplete hardware removal must be borne in mind. The fixed-angle implants in particular have given rise to a new type of complications following hardware removal. These procedures cannot therefore be recommended except for symptomatic patients. In some countries, however, implant removal operations are the second most frequently performed group of orthopaedic-traumatological procedures, so that in addition to their significance for the national economy these procedures could increase case numbers and-depending on numerous variables-also enhance revenue. When the German DRG system is used as the basis for invoicing revenues can be optimised when operations are done fast and with few complications.

\section{Keywords}

Implant removal · Fixed-angle implants . Complications · Costs $\cdot$ Revenue 

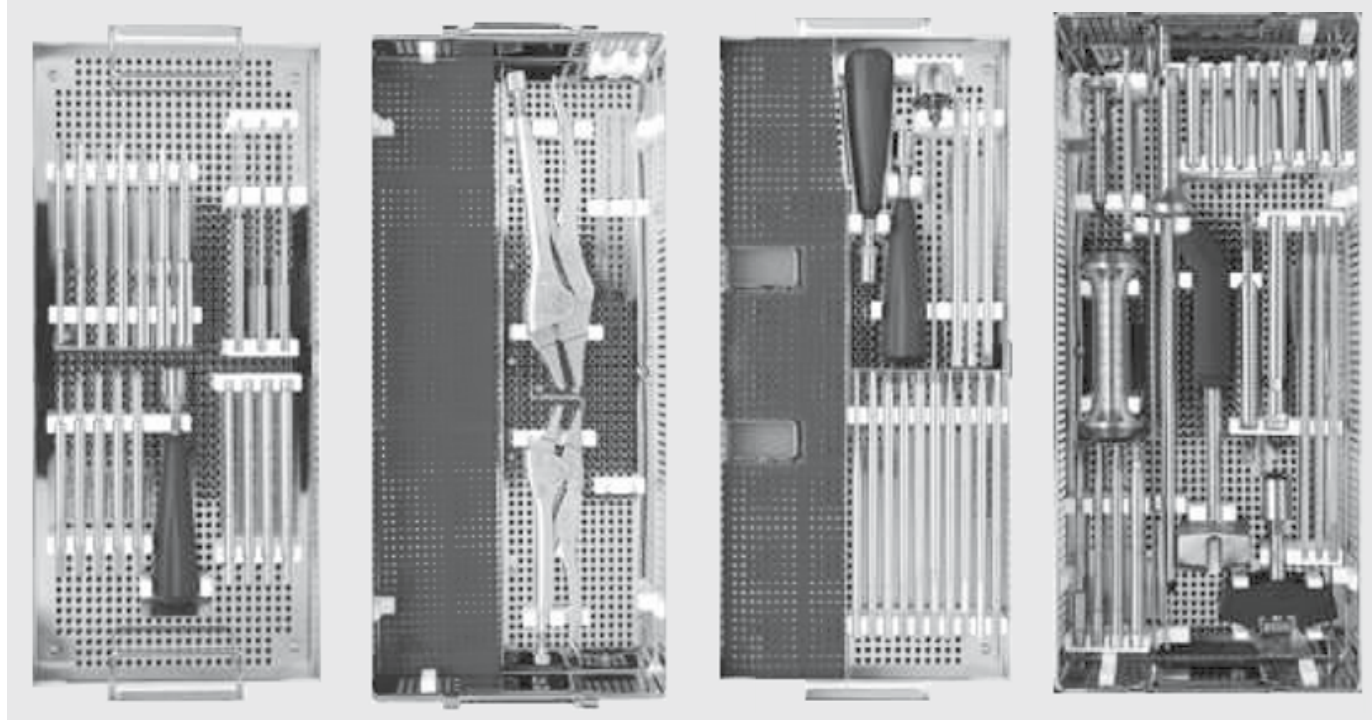

Abb. $4<$ Spezielle Extraktionssets, für jede Materialentfernung von winkelstabilen Implantaten vorzuhalten tierten) Drehmoments beim Einbringen liegt [22].

Um den aufgeführten möglichen Schwierigkeiten bei der Implantatentfernung von winkelstabilen Platten zu begegnen, werden von verschiedene Firmen Metallbohrer, Extraktionsschrauben und -bolzen sowie Hohlfräser einzeln und als fertige Sets (Extraktionsset für Standardschrauben, Extraktionsmodul für verschiedene Schraubendimensionen) angeboten. Aufgrund der vorliegenden mechanischen Untersuchungen [22], klinischer Fallpublikationen $[9,12]$ und eigener Erfahrungen [19] sollten diese speziellen Instrumente zwingend vorgehalten werden (• Abb. 4).

\section{Häufigkeit der Implantatentfernungen}

Sie variiert zwischen verschiedenen Zentren und Ländern beträchtlich - wie oben angeführt, ist die Implantatentfernung in Finnland der zweihäufigste orthopädisch-traumatologische Eingriff. Die Prävalenzen betragen 4,9\% [20] aller orthopädisch-traumatologischen Operationen (USA), 6,3\% [3] (Finnland), in einzelnen Zentren sogar $11 \%$ [4] bis $15 \%$ [3]. Somit werden bis zu etwa $80 \%$ der eingebrachten Implantate wieder entfernt [3].

\section{Kosten und Wirtschaftlichkeit}

Die durchschnittliche Häufigkeit von Implantatentfernung in Finnland mit 90 Implantatentfernungen/100.0oo Personen- jahre würde auf Deutschland umgerechnet rund 74.00o Implantatentfernungen/ Jahr (bei 82 Mio. Einwohnern) ergeben. Bei durchschnittlich vergüteten Kosten von 1622-2475 EUR für eine Implantatentfernung ergäbe das eine gesamtwirtschaftliche Belastung von 120-183 Mio. EUR.

Neben diesen gesamtgesellschaftlichen Kosten sind auch die Kosten und die potenziellen Erlöse für das Krankenhaus zu bewerten. Seit 2006 wird die Implantatentfernung im deutschen DRG-System besser abgebildet. Es existieren jetzt 3 DRG:

- die I21Z mit einer Bewertungsrelation von 0,905 (Hüftgelenk, Femur oder komplexe Eingriffe an Ellenbogengelenk und Unterarm),

- die I23A mit einer Bewertungsrelation von 0,77 (Platte an der Tibia, Wirbelsäule, Becken) und

- die I23B mit einer Bewertungsrelation von 0,593 für alle anderen Materialentfernungen.

In unserer Klinik wurde im Jahr 2006 die DRG I21Z 22-mal abgerechnet, die DRG I23A in 12 und die DRG I23B in 103 Fällen.

Die Materialentfernungen an $\mathrm{Fe}$ mur und Hüfte erbrachten insgesamt 54.450 EUR. Bei dieser DRG werden pro Eingriff durchschnittlich 2475 EUR vergütet, bei allein bereits für Operation und Anästhesie geschätzten Kosten von 1305 EUR (Daten der eigenen Klinik: durchschnittliche Schnitt-Naht-Zeit 87 min á 1o EUR und durchschnittliche Anästhesiezeit von 145 min á 3 EUR). In dieser Kalkulation sind jedoch die übrigen
Kosten (stationäre Behandlung, Röntgen, Labor...) noch nicht berücksichtigt.

Obige Zahlen gelten nur bei zügiger und komplikationsloser Operation. Wie sich mögliche Erlöse bei verlängerten Operationszeiten minimieren, sei an einem Fall einer Marknagelentfernung aus dem Femur in unserem Hause demonstriert: Bei diesem Patienten betrugen die Schnitt-Naht-Zeit 148 min und die Anästhesiezeit $197 \mathrm{~min}$, was bei gleichem Kalkulationsansatz anstelle der durchschnittlichen Eingriffkosten von 1305 EUR Kosten von 2071 EUR verursachte.

Bei der Entfernung von Femurnägeln waren in der Literatur die Nageleinbringtiefe und die Erfahrung des Operateurs [10], bei der Entfernung von Tibianägeln nur das Vorhandensein gebrochener Bolzen für die Zeitdauer der Implantatentfernung entscheidend [11].

Ob die angesprochenen Probleme und Kosten der Metallentfernung durch das Einsetzen bioresorbierbarer Implantate grundsätzlich vermieden werden können, ist zum jetzigen Zeitpunkt nicht ersichtlich. Bei ausgewählten Patientengruppen konnte für bioresorbierbaren Implantate eine geringere Reoperationsrate gezeigt werden. Eine Kostenersparnis durch eine nicht notwendige Implantatentfernung gegenüber metallischen Implantaten ist jedoch grundsätzlich von der Rate der Implantatentfernungen abhängig $[2,16]$. Diese Rentabilitätsgrenze für bioresorbierbare Implantate ist abhängig von den Kosten der Materialien, konnte aber beispielhaft für einfache (Kostenersparnis durch biore- 
sorbierbare Implantate bei einer Implantatentfernungsrate über $21 \%$ ) und komplexe Sprunggelenkfrakturen (Implantatentfernungsrate über 54\%) gezeigt werden [2].

\section{Fazit}

Eine Materialentfernung sollte mit dem Patienten individuell und gründlich abgewogen werden. Dessen exakte präoperative Aufklärung über die Risiken und den Nutzen einer Implantatentfernung ist dabei unerlässlich. Insgesamt, aber insbesondere bei winkelstabilen Implantaten, ist über eine Ausweitung des Eingriffs, Rückzugsmöglichkeiten und den Verbleib von Implantateteilen aufzuklären. Bei der Implantatentfernung, v. a. bei der Entfernung von winkelstabilen Implantaten, sind spezielle Implantatentfernungssets zwingend vorzuhalten. Falls zügig und komplikationsarm operiert wird und keine zusätzlichen Leihkosten für die speziellen Sets anfallen, können sich Implantatentfernungen Erlös steigernd auswirken. Eine Optimierung der Implantateigenschaften zur Verringerung der Verklemmung der Schraubenkopf-Platten-Verbindung und eine Intensivierung der Forschung über bioresorbierbare Implantate könnten helfen, die Komplikationen und Kosten der Implantatentfernung zu verringern.

\section{Korrespondenzadresse}

\section{Dr. M. Müller}

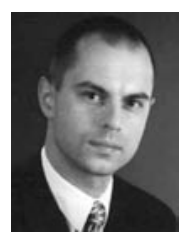

Klinik für Unfall-, Handund Wiederherstellungschirurgie, Friedrich-Schiller-Universität, Erlanger Allee 101, 07740 Jena Matthias.Mueller1@med.unijena.de

Interessenkonflikt. Der korrespondierende Autor gibt an, dass kein Interessenkonflikt besteht.

\section{Literatur}

1. Boerger TO, Patel G, Murphy JP (1999) Is routine removal of intramedullary nails justified. Injury 30 : 79-81

2. Bostman OM (1996) Metallic or absorbable fracture fixation devices. A cost minimization analysis. Clin Orthop Relat Res 329: 233-239

3. Bostman O, Pihlajamaki H (1996) Routine implant removal after fracture surgery: a potentially reducible consumer of hospital resources in trauma units. J Trauma 41: 846-849
4. Brown RM, Wheelwright EF, Chalmers J (1993) Removal of metal implants after fracture surgery indications and complications. J R Coll Surg Edinb 38: $96-100$

5. Cook SD, Renz EA, Barrack RL et al. (1985) Clinical and metallurgical analysis of retrieved internal fixation devices. Clin Orthop Relat Res 194: 236-247

6. Deluca PA, Lindsey RW, Ruwe PA (1988) Refracture of bones of the forearm after the removal of compression plates. J Bone Joint Surg Am 70: 13721376

7. Duchna HW, Nowack U, Merget R et al. (1998) [Prospective study of the significance of contact sensitization caused by metal implants]. Zentralbl Chir 123: 1271-1276

8. French HG, Cook SD, Haddad RJ (1984) Correlation of tissue reaction to corrosion in osteosynthetic devices. J Biomed Mater Res 18: 817-28

9. Georgiadis GM, Gove NK, Smith AD et al. (2004) Removal of the less invasive stabilization system. J Orthop Trauma 18: 562-564

10. Gosling T, Hufner T, Hankemeier S et al. (2004) Femoral nail removal should be restricted in asymptomatic patients. Clin Orthop Relat Res 423: 222226

11. Gosling T, Hufner T, Hankemeier S et al. (2005) [Indication for removal of tibial nails]. Chirurg 76: 789-794

12. Hamilton P, Doig S, Williamson O (2004) Technical difficulty of metal removal after LISS plating. Injury 35: 626-628

13. Hidaka S, Gustilo RB (1984) Refracture of bones of the forearm after plate removal. J Bone Joint Surg Am 66: 1241-1243

14. Highland TR, LaMont RL (1985) Deep, late infections associated with internal fixation in children. J Pediatr Orthop 5: 59-64

15. Hughes AW, Sherlock DA, Hamblen DL et al. (1987) Sarcoma at the site of a single hip screw. A case report. J Bone Joint Surg Br 69: 470-472

16. Jainandunsing JS, Van der Elst $M$, Van der Werken CC (2005) Bioresorbable fixation devices for musculoskeletal injuries in adults. Cochrane Database Syst Rev 2: CD004324

17. Miller R, Renwick SE, DeCoster TA et al. (1992) Removal of intramedullary rods after femoral shaft fracture. J Orthop Trauma 6: 460-463

18. Muller-Farber J (2003) [Removal of metal in traumatology]. Unfallchirurg 106: 653-668

19. Rausch S, Lungershausen W, Hofmann GO (2005) Die Entfernung winkelstabiler Implantate - Routine oder komplikationsträchtiges Unterfangen? Deutscher Kongress für Orthopädie und Unfallchirurgie, Berlin

20. Rutkow IM (1986) Orthopaedic operations in the United States, 1979 through 1983. J Bone Joint Surg Am 68: 716-719

21. Sanderson PL, Ryan W, Turner PG (1992) Complications of metalwork removal. Injury 23: 29-30

22. Sommer PR (2006) AxSOS versus Synthes LCP screws, insertion extraction test. Stryker International Report. Stryker Osteosynthesis 984003: LOT A1706

23. Stedtfeld HW, Landgraf $P$ (1998) [The impacted intramedullary nail. Re-discovery of an old problem with a new nail]. Unfallchirurg 101: 950-954

24. Stürmer KM (1999) Leitlinien Unfallchirurgie. Thieme, Stuttgart New York, S 12-19

25. Ward JJ, Thornbury DD, Lemons JE et al. (1990) Metal-induced sarcoma. A case report and literature review. Clin Orthop Relat Res 252: 299-306 